\title{
A Plasma Lens for High Intensity Laser Focusing
}

\author{
F. Fang*, C.E. Clayton *, N.C. Lopes ${ }^{\dagger}$, H. Ito**, K.A Marsh* and C. Joshi* \\ *UCLA Department of Electrical Engineering, Los Angeles, CA, 90095 \\ ${ }^{\dagger}$ Grupo de Lasers e Plasmas, ÊSuperior Tecnico, Lisbon \\ ${ }^{* *}$ Utsunomiya University, 7-1-2 Yoto, Utsunomiya City, Zip 321-8585, Japan
}

\begin{abstract}
A plasma lens based on a short hydrogen-filled alumina capillary discharge is experimentally characterized. For a plasma length of about $5 \mathrm{~mm}$, the focal length, measured from the plasma entrance, was $\sim 11$ to $8 \mathrm{~mm}$ for on axis densities of $\sim 2.5$ to $5 \times 10^{18} \mathrm{~cm}^{-3}$, respectively. The plasma temperature away from the walls of the $1 / 2 \mathrm{~mm}$ diameter capillary was estimated to be $\sim 8 \mathrm{eV}$ indicating that the plasma is fully ionized. Such a lens should thus be suitable for focusing very high intensity pulses. Comparisons of the measured focusing strength to that predicted by a first-order fluid model [N. A. Bobrova, et al., Phys. Rev. E 65, 016407 (2002)] shows reasonable agreement given that some of the observed plasma parameters are not predicted by this model.
\end{abstract}

Keywords: Plasma lens. focusing, high-intensity, capillary discharge, plasma temperature PACS: 52.27.-h, 52.38.-r, 52.70.Kz, 52.75.-d

\section{INTRODUCTION}

Preformed plasma channels have proven useful for guiding intense laser pulses over a few to thousand Rayleigh lengths [1]. Such channels are produced using several techniques: by novel laser-focusing geometries [2, 3, 4]; by high-voltage (HV) discharges through laser-triggered "lightning" channels [5]; or by HV discharges in a narrow (< $0.5 \mathrm{~mm}$ ) tubes (capillary discharges). Capillary discharges can be either in the wallablation mode [6] or in the gas-filled mode [7, 8]. The Rayleigh length $Z_{r}=\pi w_{o}^{2} / \lambda$ is the distance, absent the plasma channel, where the laser intensity would drop a factor of two via diffraction in vacuum. Here $w_{o}$ is the size of the laser spot at a vacuum focus and $\lambda$ is the laser wavelength. As is the case for a Graded Refractive INdex (GRIN) optical fiber, a "plasma fiber" can similarly guide a laser beam provided its refractive index is suitably "graded". Treating the plasma as a refractive medium with refractive index $\eta(r)$, the spot size evolution within the fiber is given by $\nabla_{\perp}^{2} E+k_{o}^{2} \eta(r)^{2} E=0$ where $E=E(r, z) \propto \exp \left(r^{2} / w(z)^{2}\right) \times \exp \left(i k_{o} \eta(r)\right)$. A suitable graded refractive index is one that forces the phase front of $E$ to be independent of $r$. In this case, the spot size remains constant along $z$. For an underdense plasmas, $\eta^{2}=1-n_{e}(r) / n_{c r}$ where $n_{c r}$ is the critical density for the laser. Let $n_{e}(r)=n_{e o}+\Delta n_{e} r^{2} / a^{2}$. Then, for a gaussian beam of spot size $w_{M}$, the laser will be guided at this fixed spot size if $w_{M}\left(z_{i n}\right)=\left(a^{2} /\left(\pi r_{e} \triangle n_{e}\right)\right)^{1 / 4}$ [9] and $R\left(z_{\text {in }}\right)=\operatorname{Inf}$. Here, $z_{\text {in }}$ is the entrance to the plasma fiber and $R$ is the radius of curvature of the gaussian beam. Note that $w_{M}$ is independent of $n_{e o}$ and that we have neglected relativistic and ponderomotive perturbations to the refractive index $[10,11,12]$ and subsequent focusing or guiding.

If $w\left(z_{\text {in }}\right)>w_{M}$, then the envelope of the beam will begin to focus for $z>z_{\text {in }}$ and will oscillate with a "betatron" period of $Z_{\beta} \approx \pi Z_{M}$ where $Z_{M}$ is the Rayleigh length

\author{
CP877, Advanced Accelerator Concepts: $12^{\text {th }}$ Workshop, \\ edited by M. Conde and C. Eyberger \\ (C) 2006 American Institute of Physics 978-0-7354-0378-9/06/\$23.00
}


associated with the matched spot size $w_{M}$ [13]. For a plasma-channel length of $L_{p}$, the plasma channel will act as a thick lens if $L_{p}<Z_{\beta} / 2$ [13]. In this paper we discuss preliminary experiments on such a plasma lens where $L_{p}<Z_{\beta} / 2<Z_{R}$. By propagating a well-characterized gaussian beam through this plasma lens and measuring the resultant evolution of the beam spot size, we infer $\triangle n_{e} / a^{2}$ - which is proportional to the "focusing strength" of the plasma lens-and compare the results to a simple equilibrium model [14] for the transverse plasma density distribution. The model assumes a balance between the collisional heating of the plasma due to the discharge circuit and the convective cooling to the nearby capillary walls.

\section{EXPERIMENTAL SETUP}

The experimental setup is shown in Fig. 1. Figure 1(a) shows a photograph of the $5 \mathrm{~mm}$ tall alumina capillary. The center bore (for the laser transport) has a diameter $D_{\text {cap }} \approx 550 \mu \mathrm{m}$. The top and bottom flanges are $5 \mathrm{~mm}$ in diameter and about $125 \mu \mathrm{m}$ thick. The remaining $4.75 \mathrm{~mm}$ (between the flanges) has an outer diameter of $3 \mathrm{~mm}$. There are six holes bored radially inwards at each end of the capillary for feeding $\mathrm{H}_{2}$ gas into the center bore. Figure 1(b) shows a cut-away of the mounted capillary. The capillary is press-fitted into a plastic body which also provides the $\mathrm{H}_{2}$ feed to continuously replenish the toroidal-shaped volume surrounding the $3 \mathrm{~mm}$ diameter section of the capillary. The two stainless steel electrodes are about $3 \mathrm{~mm}$ in diameter and $300 \mu \mathrm{m}$ thick with a central hole of the same size as the capillary's center bore. The brass pieces bolt onto the plastic body and make electrical contact to the electrodes through compression.

The $\mathrm{H}_{2}$ is stored at 100-500T in the reservoir which is separated from the capillary by a pulsed valve. Upon opening the valve, time is given $(\sim 100 \mathrm{~ms})$ for the gas-flow to reach steady state whereauon the high voltage (HV) discharge is initiated. Typical operating voltages were $5-30 \mathrm{kV}$ although the data presented here is at $18 \mathrm{kV}$. For this voltage, the peak current in the circuit $I_{\text {ext }} \simeq 300 \mathrm{~A}$ for a $\mathrm{HV}$ capacitor of $2 \mathrm{nF}$ and an inductance of $\approx 6 \mu \mathrm{H}$. The value of the inductor was chosen to set the rise time of the current to about 100ns which we judged fast enough to quickly heat the core of the plasma prior to substantial ion motion while not so fast as to shock-perturb the neutral density. The repetition rate of the experiment was typically $0.25 \mathrm{~Hz}$, limited by the recovery time of the vacuum. Although not presented here, both Stark broadening of the $\mathrm{H}_{\alpha}$ emission and longitudinal interferometry has shown that the peak $n_{e o}$ was much smaller than that "expected" from the reservoir pressure. We believe this is due to a low gas-conductance and associated pressure drops between the reservoir and the interior of the capillary. For example, at a reservoir pressure of $100 \mathrm{~T}$, the peak $n_{e o}$ was measured to be about $2-3 \times 10^{18} \mathrm{~cm}^{-3}, 1 / 2.5$ the "expected" reservoir pressure. A bigger factor-of-3.5 drop was also observed for the $270 \mathrm{~T}$ reservoir pressure $\left(n_{e o_{270 T}} \approx 4.5-5.5 \times 10^{18} \mathrm{~cm}^{-3}\right)$.

Figure 1(c) shows the system for imaging the laser beam exiting the capillary which consists of two lenses of focal length $f_{1}$ and $f_{2}$ separated by a distance $f_{1}+f_{2}$ with the 10-bit CCD camera located $f_{2}$ beyond the second lens. The camera was fitted with a $30 \mathrm{~nm}$ bandpass filter centered at $800 \mathrm{~nm}$, the laser wavelength. The object plane is $f_{1}$ in front of the first lens. Such a system does a scaled (maginification $=f_{2} / f_{1}$ ) double Fourier transform of the object plane onto the CCD [15]. This telescope reconstructs the 


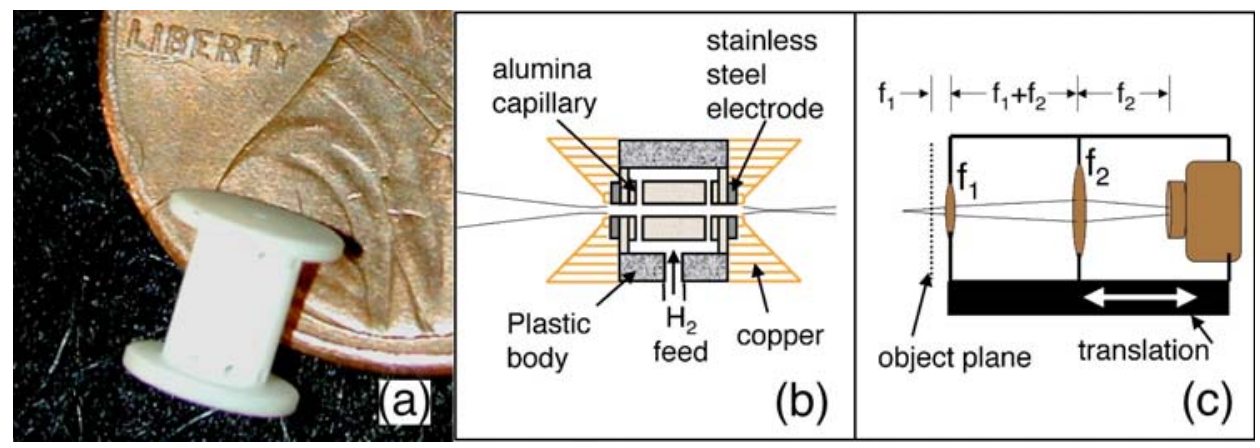

FIGURE 1. (a) A photograph of the $5 \mathrm{~mm}$ long alumina capillary outside its vacuum housing, leaning on a USA 1-cent coin. Barely discernible is the $\approx 550 \mu \mathrm{m}$ center bore and six of the twelve radial gas-feed holes located in two rings about $125 \mu \mathrm{m}$ from each flange. (b) Schematic of the mounted alumina capillary (see text for details). (c) Schematic of the imaging system. A single 10-bit image was acquired for each shot.

phase fronts as well as the intensity. Typically, the imaging system was scanned in $1 \mathrm{~mm}$ steps over a $10 \mathrm{~mm}$ range starting with the object plane coincident with the exit of the capillary.

The laser beam was obtained by sending a small portion $(<<1 \mathrm{~mJ})$ of the output of a Ti-Sapph regenerative amplifier through a moderate spatial filter. Residual diffraction rings after the spatial filter were eliminated by propagating the beam an additional $8 \mathrm{~m}$ with a mild focus at the $\sim 5 \mathrm{~m}$ mark. This gave a near-diffraction-limited beam at the location of the experiment. The beam was focused through a vacuum window with a 500mm focal length lens (not shown) to a spot size of about $60 \mu \mathrm{m}$ at the entrance to the capillary. Two additional Fourier transform lenses (not shown) relayed the capillary exit plane (with 1:1 magnification) to a position outside the vacuum on the far side of the chamber (not shown). For such a large incoming spot size, $Z_{r} \approx 13 \mathrm{~mm}$ so that, without the discharge, the laser will emerge cleanly from the exit of the capillary allowing for an accurate in situ measurement of the incoming beam parameters.

\section{RESULTS}

Figure 2 shows the measured spot size beyond the capillary exit for the case of no plasma (vacuum, black diamonds) and for $\mathrm{H}_{2}$ reservoir pressures of 100T (Fig. 2(a)) and 270T (Fig. 2(b)) shown as red circles. The injection time $t_{i n j}$ of the $\sim 200 \mathrm{ps}-$ long laser pulse into the capillary was about 60ns (40ns) for the low (high) pressure case. One can see that for the particular low-pressure scan of Fig. 2(a), the laser was still converging as it entered the capillary while for the high-pressure case of 2(b) the waist was just at the capillary entrance. The shot-to-shot variation in the vacuum data, observed as "breathing" of the incoming beam, was probably due to air currents in the laboratory although most (but not all) of the $\sim 8 \mathrm{~m}$ path was enclosed in $75 \mathrm{~mm}$ diameter plastic tubing. For the low-pressure, plasma-on data (red circles in 2(a)), the shot-to-shot variation is slightly larger. Examination of the images suggest that the "breathing" of the input beam caused the beam to arrive off-center in the discharge which caused a slight 

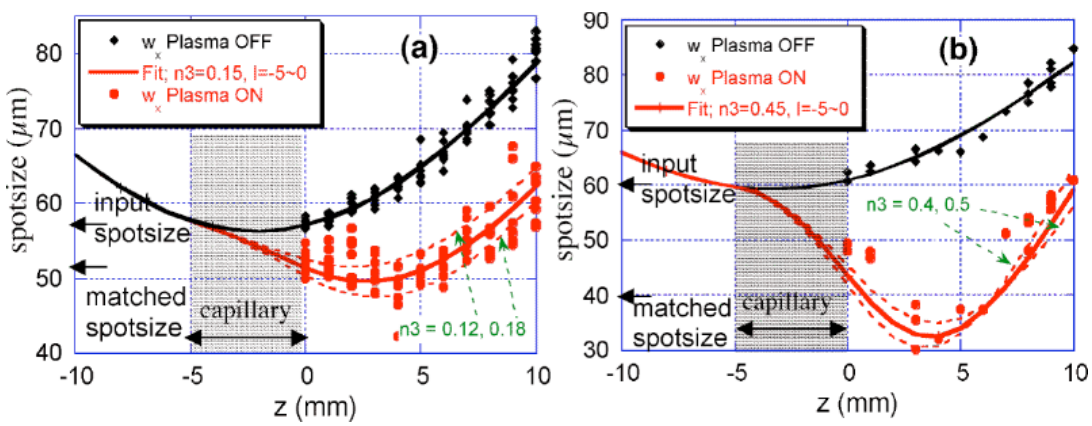

FIGURE 2. Laser beam spot-soze measurements in vacuum (black diamonds) and with the plasma on (red circles) for reservoir pressures of $100 \mathrm{~T}$ (a) and 270T (b). In both (a) and (b), the solid black lines (solid red lines) are free-space gaussian-beam propagation fits to the plasma off (plasma on) data. The dashed green lines indicate reasonable bounds to the fits (see next Section). Also indicated by the black arrows are the location of the capillary, the input spot sizes, and the calculated matched-spot sizes based on the fits.

transverse kick to the beam after the capillary. Nevertheless, every data point can be plotted as this effect was minimal.

In contrast to the low-pressure data, the high pressure data of Fig. 2(b) had to be "filtered", eliminating events that were very far off axis. Concomitant with the increased focusing strength is an increase sensitivity to off-axis injection. Further complicating the experiment was also jitter in the discharge timing (as discussed below) and possible shot-to-shot asymmetries in the plasma, as a post mortem of the experiment revealed a slightly misaligned stainless steel electrode.

The vacuum data was fit to a free-space gaussian model giving the "complex gaussian parameter" $1 / q(z)=1 / R(z)-i \lambda /\left(\pi w(z)^{2}\right)$ at the entrance to the capillary. The resulting $1 / q\left(z_{\text {in }}\right)$ was used as the initial condition for the gaussian "ABCD" matricies describing the plasma and the free space beyond. There are two main fit parameters which can be adjusted to find a spot-size evolution matching that observed in Fig. $2 ; L_{p}$ and $\triangle n_{e} / a^{2}$, the latter being the proportional to the second derivative of the $n_{e}(r)$. For simplicity, we fixed $L_{p}$ at $5 \mathrm{~mm}$ and adjusted the curvature of the plasma channel which appears in the sine-cosine-like ABCD matrix for a GRIN optic.

The results of the fitting procedure are shown in Table 1 where $n_{3}$ is defined as the change in density in units of $10^{18} \mathrm{~cm}^{-3}$ at $r=100 \mu \mathrm{m}$. For example, in the fit for the $270 \mathrm{~T}$ case, $n_{3} \approx 0.45$ so that the inferred parabolic shape of the radial density profile is such that, at $r=100 \mu \mathrm{m}$, the density increases from the on-axis density $n_{e o}$ to $n_{e o}+0.45 \times 10^{18} \mathrm{~cm}^{-3}$ over a distance of $100 \mu \mathrm{m}$. For the $100 \mathrm{~T}$ case, the observed focusing is much weaker; $n_{3} \approx 0.15$ which is smaller than the $270 \mathrm{~T}$ case as be expected.

The third column in Table 1 gives $n_{3}$ for a reservoir pressure of 100T, but for $t_{i n j}=$ 140ns. The focusing (not shown) at this late time is $\sim$ half that observed at the same pressure but with $t_{i n j}=60 \mathrm{~ns}$. The time $t_{i n j}$ of 60ns was found to be optimum for 100T in terms of focusing strength of the plasma channel, as shown in Figure 3. In Fig. 3, the object plane for the imaging telescope was set to $\mathrm{z}=3 \mathrm{~mm}$ beyond the capillary exit and $t_{i n j}$ was scanned. The circles (diamonds) show the spot size vs $t_{i n j}$ for a reservoir pressure of 100T (270T). We conclude that the "guiding window" for the lower pressure 
TABLE 1. Plasma channel depth from free-space gaussianpropagation fits to the measured plasma-on spot size evolution for 100T and 270T at the noted injection times and estimates of the channel depth from a simple Local Equilibrium Model (LEM). The depth is in units of $n_{3}$ which is the change in density $100 \mu \mathrm{m}$ off axis.

\begin{tabular}{lrrr}
\hline & 100T & 270T & 100T \\
\hline$t_{\text {inj }}(\mathrm{ns})$ & 60 & 40 & 140 \\
$n_{3}$ from fit $10^{18} \mathrm{~cm}^{-3}$ & $0.15 \pm 0.03$ & $0.45 \pm 0.05$ & $0.08 \pm 0.02$ \\
$n_{3}$ from LEM $10^{18} \mathrm{~cm}^{-3}$ & 0.35 & 0.94 & 0.35 \\
\hline
\end{tabular}

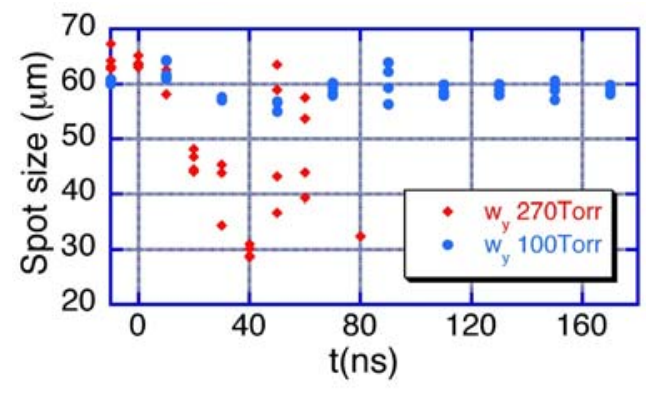

FIGURE 3. Measured variation of the plasma-on spot size for reservoir pressures of 270T (red diamonds) and 100T (blue circles) with the telescope set to image a plane located $3 \mathrm{~mm}$ beyond the capillary exit. Times less than zero are prior to firing the discharge. Note that the spot size change for $100 \mathrm{~T}$ is artificially attenuated since the object plane was $2-3 \mathrm{~mm}$ in front of the "best focus" for 100T.

is wider in time than that for the higher pressure and that it occurs later in the evolution of the plasma. We also notice that, for the strong-focusing case of 270T, there is large "jitter" in the spot size. We believe that this is due to the shortness of the guiding window coupled with finite jitter of the discharge circuit at the operating voltage of $18 \mathrm{kV}$. The measured peak-to-peak jitter in the discharge was only about 5-7ns at $18 \mathrm{kV}$ suggesting that the guiding window is not much longer than this.

The short $(<10-20 \mathrm{~ns})$ windows for clear focusing shown in Fig. 3 along with the small $n_{3}$ at 100T and $t_{i n j}$ of 140ns suggests that the plasma-channel depth drops for later times. As discussed in the next Section, $T_{e}$ does not appear to drop. Thus it is likely that the drop in $n_{3}$ is due to a falling $n_{e o}$. This is not surprising given the small (10:1) aspect ratio of the capillary and the inevitability of longitudinal flow of the plasma out the ends of the capillary.

\section{DISCUSSION}

\section{Comparison to a simple fluid model}

A simple, quasi-steady-state plasma-fluid model [14] was used to estimate $n_{3}$ based on $I_{e x t}$ and $D_{c a p}$. This "local equilibrium model" (LEM) neglects the transient ionization phase of the early part of the discharge. It is assumed that the electron temperature $T_{e}$ is 
always in equlibrium between ohmic heating, given by $\eta_{S p} J^{2}$ and the radial divergence of the heat flux $q_{e}=-\kappa_{e} d T_{e} / d r$ such that

$$
-\frac{1}{r} \frac{d}{d r}\left(r \kappa_{e} \frac{d T_{e}}{d r}\right)=\eta_{S p} J^{2}=\eta_{S p} \frac{I_{e x t}^{2}}{A_{p}^{2}} .
$$

Here, $\eta_{S p} \approx \frac{\pi e^{2} m^{1 / 2}}{\left(k T_{e}\right)^{3 / 2}} \ln \Lambda$ is the Spitzer resistivity [16], $\kappa_{e} \approx \frac{2.4\left(k T_{e}\right)^{5 / 2}}{\left(2 \pi m_{e}\right)^{1 / 2} e^{4} \ln \Lambda}$ is the electron thermal conductivity, and we have used $J A_{p}=I_{\text {ext }}$ where $A_{p}=\pi D_{\text {cap }}^{2} / 4$.

By using the boundary conditions $T_{e}\left(r=D_{\text {cap }} / 2\right)=0$ and $d T_{e} / d r(r=0)=0$, Eq. (1) can be numerically solved yielding the radial temperature profile $T_{e}\left(r, I_{\text {ext }}, D_{\text {cap }}\right)$. Note that Eq. (1) is essentially independent of density in this first-order model. For $I_{e x t}=350 \mathrm{~A}, T_{e}(r=0) \approx 2.5 \mathrm{eV}$. (We believe that this predicted temperature is too low by a factor of 3 , as discussed below.) The radial variation of the plasma density is obtained by using pressure balance; $n_{e}(r) k T_{e}(r)=n_{e}(0) k T_{e}(0) \approx n_{e o} k T_{e}(0)$. This is where the density dependence arises: for a given $I_{e x t}$ and $D_{c a p}$, the on-axis temperature is determined and, for example, if one increases $n_{e o}$, the rate of increase of $n_{e}(r)$ must also increase to maintain pressure balance. The focusing strength $n_{3}$ obtained from this calculated $n_{e}(r)$ is shown in Table 1 . Recalling that the density inside the capillary is really $1 / 2.5$ at 100 Torr(or 1/3.5 at 270 torr) the reservoir pressure, we should divide the LEM numbers by these factor. The "agreement" with these modified LEM numbers and experiment is reasonable.

\section{Plasma resistance measurement and estimates of $T_{e}(t)$}

The "predicted" temperature from the fluid model above does not agree with that derived from a basic measurement of the circuit resistance of the plasma discharge. A HV probe was placed across the plasma electrodes (the brass pieces in Fig. 1(b)) to measure the voltage across the plasma $V_{p}$ and a current monitor measured $I_{\text {ext }}$ on the HV lead to the capillary. The electric field in the plasma can be expressed as $E_{p} \approx V_{p} / L_{p}=\eta_{S p} J$. Defining the effective plasma area as $A_{e f f}=\alpha \pi D_{c a p}^{2} / 4$ and letting $J=I_{\text {ext }} / A_{\text {eff }}$, we can deduce the Spitzer resistivity as $\eta_{S p}=\frac{A_{\text {eff }}}{L_{p}} \frac{V_{p}}{I_{\text {ext }}}$. Here, $\alpha$ is the fraction of capillary cross-sectional area containing the bulk of the current flow and $V_{p} / I_{\text {ext }}$ is the measured resistance $R_{p}$ at the time of guiding. Since $T_{e}(r)$ peaks on axis, the plasma current flows in a central channel which is somewhat narrower than $D_{\text {cap }}$. We take the diameter of the current channel as the FWHM of the temperature profile raised to the power of $3 / 2$; the scaling of the Spitzer conductivity. This gives $\alpha \approx 0.7$ and, for $R_{p} \approx 2 \Omega$, we get $T_{e} \sim 8 \mathrm{eV}$, about $3 \times$ larger than the LEM model discussed above. The discrepency may be revealed by examining the oscilloscope traces for $V_{p}$ and $I_{\text {ext }}$ (not shown). Prior to "breakdown", the voltage across the electrodes increases up to a few $\mathrm{kV}$ and the measured current is the displacement current. Once breakdown occurs, there is a finite time where $V_{p}$ is falling and $I_{e x t}$ is rising. During this "breakdown transient", power $\left(V_{p}(t) \times I_{\text {ext }}(t)\right)$ is dumped into the plasma, raising its temperature beyond that 
expected from the LEM model where the initial density is $10^{13} \mathrm{~cm}^{-3}$ and the heating is adiabatic.

\section{CONCLUSIONS}

A plasma lens based on a short hydrogen-filled alumina capillary discharge has been experimentally characterized. For a plasma length of about $5 \mathrm{~mm}$, the focal length, measured from the plasma entrance, was $\sim 11$ to $8 \mathrm{~mm}$ for on axis densities of $\sim 2.5$ to $5 \times 10^{18} \mathrm{~cm}^{-3}$, respectively. The plasma temperature away from the walls of the $1 / 2 \mathrm{~mm}$ diameter capillary was estimated to be $\sim 8 \mathrm{eV}$ indicating that the plasma is fully ionized. Such a lens should thus be suitable for focusing very high intensity pulses. Comparisons of the measured focusing strength to that predicted by first-order fluid model shows reasonable agreement.

\section{ACKNOWLEDGMENTS}

The authors would like to thank Yasushi Nishida of Utsunomiya University for his initial support to this work. This work was supported by Grant no. DE-FG52-03NA00138, DEFG02-92ER40727, and IST Grant No. POCI/58776 (Ack. FCT-Portugal).

\section{REFERENCES}

1. E. Esarey, P. Sprangle, J. Krall, and A. Ting, IEEE Trans. Plasma Sci. 24, 252-288 (1996).

2. C. G. D. III, and H. M. Milchberg, Phys. Rev. Lett. 71, 2409 (1993).

3. P. Volfbeyn, E. Esarey, and W. P. Leemans, Phys. Plasmas 6, 2269 (1999).

4. E. W. Gaul, S. P. L. Blanc, A. R. Rundquist, R. Zgadzaj, H. Langhoff, and M. C. Downer, Appl. Phys. Lett. 77, 4112 (2000).

5. N. C. Lopes, G. Figueira, L. O. Silva, J. M. Dias, R. Fonseca, L. Cardoso, C. Russo, C. Carias, G. Mendes, J. Vieira, and J. T. Mendonca, Phys. Rev. E 68, 035402(R) (2003).

6. Y. Ehrlich, C. Cohen, D. Kaganovich, A. Zigler, R. F. Hubbard, P. Sprangle, and E. Esarey, J. Opt. Soc. Amer. B. 15, 2416 (1998).

7. A. Butler, D. J. Spence, and S. M. Hooker, Phys. Rev. Lett. 89, 185003 (2002).

8. T. Hosokai, M. Kando, H. Dewa, H. Kotaki, S. Kondo, N. Hasegawa, K. Nakajima, and K. Horioka, Opt. Lett. 25, 10-12 (2000).

9. Y. Ehrlich, C. Cohen, A. Zigler, P. S. J. Krall, , and E. Esarey, Phys. Rev. Lett. 77, 4186 (1996).

10. C. Ren, B. J. Duda, R. G. Hemker, W. B. Mori, T. Katsouleas, T. M. Antonsen, and P. Mora, Phys. Rev. E 63, 026411 (2001).

11. E. Esarey, C. B. Schroeder, B. A. Shadwick, J. S. Wurtele, and W. P. Leemans, Phys. Rev. Lett. 84, $3081(2000)$.

12. W. Lu, C. Huang, M. Zhou, M. Tzoufras, F. S. Tsung, W. B. Mori, and T. Katsouleas, Phys. Plasmas 13, 056709 (2006).

13. R. F. Hubbard, B. Hafizi, A. Ting, D. Kaganovich, P. Sprangle, and A. Zigler, Phys. Plasmas 9, 1431-1442 (2002).

14. N. A. Bobrova, A. A. Esaulov, J. I. Sakai, P. V. Sasorov, D. J., Spence, A. Butler, S. M. Hooker, and S. V. Bulanov, Phys. Rev. E 65, 016407 (2002).

15. J. Goodman, Introduction to Fourier Optics, McGraw-Hill, New York, 1968.

16. J. D. Huba, NRL Plasma Formulary, Navel Research Laboratory, 2002, nrl/pu/6790-02-450 edn. 